\title{
Kann ich die abrechnen?
}

Frau Dr. med. A. H., Internistin, Köln Ich bin fachärztlich tätig und erhalte auch Überweisungen von Fachärzten. Ich pflege in diesen Fällen auch den Hausärzten eine Kopie des Briefes an den Facharzt zukommen zu lassen. Kann ich die Kopie an den Hausarzt verrechnen, auch wenn die Überweisung vom Facharzt kommt?

Antwort: Es gibt im EBM eine GOP 01602 für die Mehrfertigung (z. B. Kopie) eines Berichtes oder Briefes nach den GOP 01 600, 01601 an den Hausarzt $1,23 €$. Bei der Berechnung der GOP 01602 ist auf dem Behandlungsausweis die Arztabrechnungsnummer oder der Name des Hausarztes anzugeben.

Die GOP 01602 für die Kopie eines Berichtes oder Briefes an den Hausarzt ist nur berechnungsfähig, wenn bereits ein Bericht oder Brief an einen anderen Arzt erfolgt ist. Zusätzlich kann selbstverständlich noch Porto nach den GOP 40 120, Kostenpauschale für die Versendung bzw. den Transport von Briefen und/oder schriftlichen Unterlagen bis $20 \mathrm{~g}$ (z. B. im Postdienst Standardbrief) oder für die Übermittlung eines Telefax, $€ 0,55$, in Ansatz gebracht werden. Beide GOP können auch dann verrechnet werden, wenn die "Kopie" kostensparend ohne Ausdruck direkt vom PC versandt wird.

\section{Ich mache in meiner Praxis Infusionen}

\section{Welche GOP gilt?}

Dr. med. E. Sch., Facharzt für Allgemeinmedizin, KV Niedersachsen Ich mache immer wieder Infusionen in der Praxis. Wie sind diese abrechenbar?

Antwort: Es gibt im EBM zwei Positionen für Infusionen: Die GOP 02100 Infusion, intravenös und/oder in das Knochenmark, mittels Portsystem, intraarteriell, Dauer mindestens zehn Minuten, 5,61 € und die GOP 02 101, Infusions-Therapie u. a. mit Zytostatika, Virustatika, Antimykotika und/oder Antibiotika bei einem Kranken mit konsumierender Erkrankung, Dauer mindestens 60 Minuten, 15,60€.

Im normalen Praxisalltag fallen diese GOP in die Versichertenpauschale und sind im EBM nicht gesondert abrechenbar. Aus betriebswirtschaftlichen Gründen sollte man daher

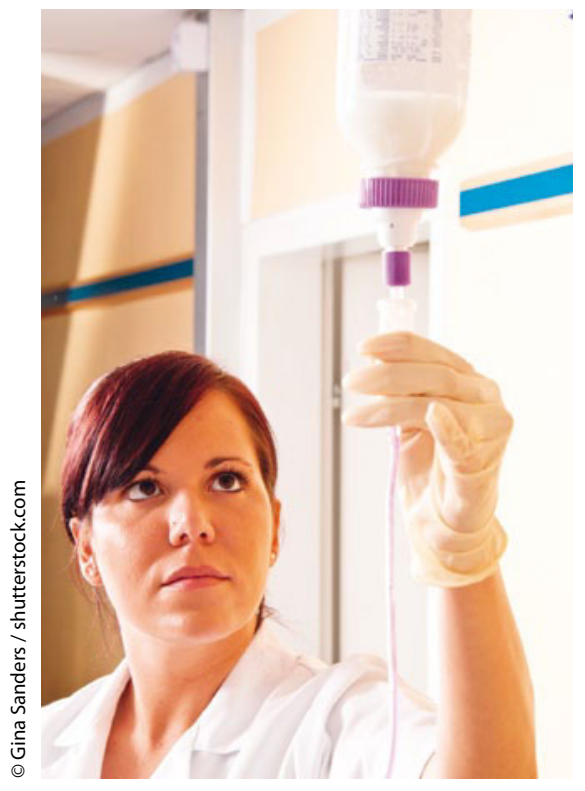

ernsthaft überlegen, ob man diese Leistung im Rahmen der GKV überhaupt erbringt, außer bei absoluter Indikation oder im Notfall. Allerdings gibt es eine Ausnahme: Im Notfalldienst, also neben der GOP 01 210, Notfallpauschale im organisierten Not(fall-) dienst und den Notfallkonsultationspauschalen, sind die Positionen gesondert abrechenbar.

In der GOÄ gibt es diese Ausschlüsse nicht. Hier kommen je nach Leistung die GOP 270 bis 279 infrage. Wird eine dieser Leistungen der GOÄ erbracht, ist sie auch vom Allgemeinarzt uneingeschränkt abrechenbar.

4 Infusionen in der Praxis sind betriebswirtschaftlich unrentabel. 